EPJ Web of Conferences 67, 02015 (2014)

DOI: $10.1051 /$ epjconf/20146702015

(c) Owned by the authors, published by EDP Sciences, 2014

\title{
The discontinuous Galerkin method for the numerical simulation of compressible viscous flow
}

\author{
Jan Česenek ${ }^{1, a}$ \\ ${ }^{1}$ Aerospace Research and Test Establishment, Beranových 130, 19905 Praha - Letňany, Czech Republic
}

\begin{abstract}
In this paper we deal with numerical simulation of the compressible viscous flow. The mathematical model of flow is represented by the system of non-stationary compressible Navier-Stokes equations. This system of equations is discretized by the discontinuous Galerkin finite element method in space and in time using piecewise polynomial discontinuous approximations. We present some numerical experiments to demonstrate the applicability of the method using own-developed code.
\end{abstract}

\section{Introduction}

During the last decade the discontinuous Galerkin finite element method (DGM), which is based on piecewise polynomial discontinuous approximations of the sought solution, became very popular in the field of numerical simulation of the fluid flow. In this paper we present the DGM for numerical simulation of compressible viscous flow around a moving airfoil. A solid airfoil with two degrees of freedom performs rotation around an elastic axis and oscillations in the vertical direction. Compressible viscous flow is described by the system of the Navier-Stokes equations. Due to the time dependent domain the Navier-Stokes equations are transformed in the ALE (Arbitrary LagrangianEulerian) formulation. We consider two approaches to the discretization using the DGM, which differ in the time discretization. First we apply the backward difference formula (BDF) to aproximate the ALE derivative. Second, the full space-time discontinuous Galerkin method (ST-DG) is employed. The discrete flow problem is coupled with the system of ordinary differential equations describing airfoil vibrations. Results of numerical simulation are presented.

\section{ALE formulation of the Navier-Stokes equations}

We consider compressible viscous flow in a bounded domain $\Omega(t) \subset R^{d}, d=2,3$, depending on time $t \in[0, T]$. We assume that the boundary $\Omega(t)$ consists of three disjoint parts $\partial \Omega(t)=\Gamma_{I} \cup \Gamma_{O} \cup \Gamma_{W}(t)$, where $\Gamma_{I}$ is the inlet, $\Gamma_{O}$ is the outlet and $\Gamma_{W}(t)$ is impermeable wall, whose parts may move.

The time dependence of the domain is taken into account with the aid of a regular one-to-one ALE mapping

$$
\mathcal{A}_{t}: \Omega(0) \rightarrow \Omega(t) .
$$

We define the ALE velocity $z$ by the relations

$$
\begin{aligned}
\tilde{\boldsymbol{z}}(\boldsymbol{X}, t) & =\frac{\partial}{\partial t} \mathcal{A}_{t}(\boldsymbol{X}), \quad t \in[0, T], \boldsymbol{X} \in \Omega(0), \\
\boldsymbol{z}(\boldsymbol{x}, t) & =\tilde{\boldsymbol{z}}\left(\mathcal{A}_{t}^{-1}(\boldsymbol{x}), t\right), \quad t \in[0, T], \boldsymbol{x} \in \Omega(t)
\end{aligned}
$$

and the ALE derivative of a vector function $\mathbf{w}=\mathbf{w}(\boldsymbol{x}, t)$ defined for $\boldsymbol{x} \in \Omega(t)$ and $t \in[0, T]$ :

$$
\frac{D^{\mathcal{A}}}{D t} \mathbf{w}(\boldsymbol{x}, t)=\frac{\partial \tilde{\mathbf{w}}}{\partial t}(\boldsymbol{X}, t)
$$

where

$$
\tilde{\mathbf{w}}(\boldsymbol{X}, t)=\mathbf{w}\left(\mathcal{A}_{t}(\boldsymbol{X}), t\right), \boldsymbol{X} \in \Omega(0), x=\mathcal{A}_{t}(\boldsymbol{X}) .
$$

Then the system of the Navier-Stokes equations describing the compressible viscous flow can be written in the ALE form

$$
\frac{D^{\mathcal{A}} \mathbf{w}}{D t}+\sum_{s=1}^{2} \frac{\partial \mathbf{g}_{s}(\mathbf{w})}{\partial x_{s}}+\mathbf{w d i v} \boldsymbol{z}=\sum_{s=1}^{2} \frac{\partial \mathbf{R}_{s}(\mathbf{w}, \nabla \mathbf{w})}{\partial x_{s}},
$$

where for $i, j=1, \ldots, d$ we have

$$
\begin{aligned}
\mathbf{w} & =\left(w_{1}, \ldots, w_{d+2}\right)^{T}=\left(\rho, \rho v_{1}, \ldots, \rho v_{d}, E\right)^{T} \in R^{d+2}, \\
\mathbf{f}_{i}(\mathbf{w}) & =\left(f_{i, 1}, \ldots, f_{i, d+2}\right)^{T} \\
& =\left(\rho v_{i}, \rho v_{1} v_{i}+\delta_{1 i} p, \ldots, \rho v_{d} v_{i}+\delta_{d i} p,(E+p) v_{i}\right)^{T}, \\
\mathbf{g}_{i}(\mathbf{w}) & =\mathbf{f}_{i}(\mathbf{w})-z_{i} \mathbf{w}, \\
\mathbf{R}_{i}(\mathbf{w}, \nabla \mathbf{w}) & =\left(R_{i, 1}, \ldots, R_{i, d+2}\right)^{T} \\
& =\left(0, \tau_{i 1}^{V}, \ldots, \tau_{i d}^{V}, \sum_{s=1}^{d} \tau_{i s}^{V} v_{s}+k \frac{\partial \theta}{\partial x_{i}}\right)^{T}, \\
\tau_{i j}^{V} & =\lambda \operatorname{div} \boldsymbol{v} \delta_{i j}+2 \mu d_{i j}(\boldsymbol{v}), \quad d_{i j}(\boldsymbol{v})=\frac{1}{2}\left(\frac{\partial v_{i}}{\partial x_{j}}+\frac{\partial v_{j}}{\partial x_{i}}\right) .
\end{aligned}
$$

We use the following notation: $\boldsymbol{v}=\left(v_{1}, \ldots, v_{d}\right)$ - velocity, $\rho$ - density, $p$ - pressure, $\theta$ - absolute temperature, $E$ - total energy, $\gamma$ - Poisson adiabatic constant, $c_{v}$ - specific heat at constant volume, $\mu>0, \lambda=-2 \mu / 3$ - viscosity coefficients, $k$ - heat conduction coefficient. The above system is completed by the thermodynamical relations

$$
p=(\gamma-1)\left(E-\frac{\rho|\boldsymbol{v}|^{2}}{2}\right), \quad \theta=\frac{1}{c_{v}}\left(\frac{E}{\rho}-\frac{1}{2}|\boldsymbol{v}|^{2}\right)
$$

and is equipped with the initial condition

$$
\mathbf{w}(x, 0)=\mathbf{w}^{0}(x), x \in \Omega(0),
$$

\footnotetext{
a e-mail: cesenek@vzlu.cz
} 
and the following boundary conditions
a) $\left.\rho\right|_{\Gamma_{l}}=\rho_{D}$,
b) $\left.\boldsymbol{v}\right|_{\Gamma_{I}}=\boldsymbol{v}_{D}=\left(v_{D 1}, \ldots, v_{D d}\right)^{T}$,
c) $\sum_{i, j=1}^{d} \tau_{i j}^{V} n_{i} v_{j}+k \frac{\partial \theta}{\partial n}=0 \quad$ on $\Gamma_{I}$,
d) $\left.\boldsymbol{v}\right|_{\Gamma_{W}(t)}=z_{D}=$ velocity of a moving wall,
e) $\left.\frac{\partial \theta}{\partial n}\right|_{\Gamma_{W}(t)}=0$
f) $\sum_{i=1}^{d} \tau_{i j}^{V} n_{i}=0, j=1, . ., d, \quad$ on $\Gamma_{O}$,
g) $\left.\frac{\partial \theta}{\partial n}\right|_{\Gamma_{O}}=0$

with given data $\mathbf{w}^{0}, \rho_{D}, \boldsymbol{v}_{D}, \boldsymbol{z}_{D}$. It is possible to show that $\mathbf{f}_{s}(\alpha \mathbf{w})=\alpha \mathbf{f}_{s}(\mathbf{w})$ for $\alpha>0$. This property implies that

$$
\mathbf{f}_{s}(\mathbf{w})=\mathbf{A}_{s}(\mathbf{w}) \mathbf{w}, \quad s=1, . ., d,
$$

where

$$
\mathbf{A}_{s}(\mathbf{w})=\frac{D \mathbf{f}_{s}(\mathbf{w})}{D \mathbf{w}}, \quad s=1, . ., d,
$$

are the Jacobi matrices of the mapping $\mathbf{f}_{s}$. Expression of the matrices $\mathbf{A}_{s}$ can be found in [4]. The viscous terms $\mathbf{R}_{s}(\mathbf{w}, \nabla \mathbf{w})$ can be expressed in the form

$$
\mathbf{R}_{s}(\mathbf{w}, \nabla \mathbf{w})=\sum_{k=1}^{d} \mathbf{K}_{s, k}(\mathbf{w}) \frac{\partial \mathbf{w}}{\partial x_{k}}, \quad s=1, . ., d,
$$

where $\mathbf{K}_{s, k}(\mathbf{w}) \in \boldsymbol{R}^{(d+2) \times(d+2)}$ are matrices depending on $\mathbf{w}$. Expression of the matricies $\mathbf{K}_{s, k}(\mathbf{w})$ can be found in [3].

\section{Discretization}

\subsection{Space discretization of the flow problem}

By $\Omega_{h}(t)$ we denote polygonal $(\mathrm{d}=2)$ or polyhedral $(\mathrm{d}=3)$ approximation of the domain $\Omega(t)$. Let $\mathcal{T}_{h}(t)$ be a partition of the domain $\Omega_{h}(t)$ into finite number of closed elements with mutually disjoint interiors such that $\overline{\Omega_{h}(t)}=$ $\bigcup_{K \in \mathcal{T}_{h}(t)} K$. In 2D problems, we usually choose $K \in \mathcal{T}_{h}(t)$ as triangles or quadrilaterals. In $3 \mathrm{D}, K \in \mathcal{T}_{h}(t)$ can be, e.g., tetrahedrons, prisms or hexahedrons. By $\mathcal{F}_{h}(t)$ we denote the system of all faces of all elements $K \in \mathcal{T}_{h}(t)$. Further, we introduce the set of boundary faces $\mathcal{F}_{h}^{B}(t)=$ $\left\{\Gamma \in \mathcal{F}_{h}(t) ; \Gamma \subset \partial \Omega_{h}(t)\right\}$ and the set of inner faces $\mathcal{F}_{h}^{I}(t)=\mathcal{F}_{h}(t) \backslash \mathcal{F}_{h}^{B}(t)$. Each $\Gamma \in \mathcal{F}_{h}(t)$ is associated with a unit normal vector $\boldsymbol{n}_{\Gamma}$. For $\Gamma \in \mathcal{F}_{h}^{B}(t)$ the normal $\boldsymbol{n}_{\Gamma}$ has the same orientation as the outer normal to $\partial \Omega_{h}(t)$. For each $\Gamma \in \mathcal{F}_{h}^{I}(t)$ there exist two neighbouring elements $K_{\Gamma}^{L}, K_{\Gamma}^{R} \in \mathcal{T}_{h}(t)$ such that $\Gamma \subset \partial K_{\Gamma}^{L} \cap \partial K_{\Gamma}^{R}$. We use the convention that $K_{\Gamma}^{R}$ lies in the direction of $\boldsymbol{n}_{\Gamma}$ and $K_{\Gamma}^{L}$ lies in the opposite direction to $\boldsymbol{n}_{\Gamma}$. If $\Gamma \in \mathcal{F}_{h}^{B}(t)$, then the element adjacent to $\Gamma$ will be denoted by $K_{\Gamma}^{L}$.

We shall look for an approximate solution of the problem in the space of piecewise polynomial functions

$\mathbf{S}_{h}^{p}(t)=\left(S_{h}^{p}(t)\right)^{d+2}, \quad S_{h}^{p}(t)=\left\{v ;\left.v\right|_{K} \in P^{p}(K), \forall K \in \mathcal{T}_{h}(t)\right\}$, where $p>0$ is an integer and $P^{p}(K)$ denotes the space of all polynomials on $K$ of degree $\leq p$. A function $\Phi \in \mathbf{S}_{h}^{p}(t)$ is, in general, discontinuous on interfaces $\Gamma \in \mathcal{F}_{h}^{I}(t)$. By $\Phi_{\Gamma}^{L}$ and $\Phi_{\Gamma}^{R}$ we denote the values of $\Phi$ on $\Gamma$ considered from the interior and the exterior of $K_{\Gamma}^{L}$, respectively, and set

$$
\begin{aligned}
& \langle\Phi\rangle_{\Gamma}=\frac{1}{2}\left(\Phi_{\Gamma}^{L}+\Phi_{\Gamma}^{R}\right), \\
& {[\Phi]_{\Gamma}=\Phi_{\Gamma}^{L}-\Phi_{\Gamma}^{R},}
\end{aligned}
$$

which denotes average and jump of $\Phi$ on $\Gamma$.

The discrete problem is derived in the following way: For arbitrary $t \in[0, T]$ we can multiply the system by a test function $\mathbf{S}_{h}^{p}(t)$, integrate over $K \in \mathcal{T}_{h}(t)$, apply Green's theorem, sum over all elements $K \in \mathcal{T}_{h}(t)$, use the concept of the numerical flux and introduce suitable terms mutually vanishing for a regular exact solution. Moreover, we carry out a suitable partial linearization of nonlinear terms.

In order to evaluate the integrals over $\Gamma \in \mathcal{F}_{h}(t)$ in inviscid term we use the approximation

$$
\int_{\Gamma} \mathbf{H}\left(\mathbf{w}_{\Gamma}^{L}, \mathbf{w}_{\Gamma}^{R}, \boldsymbol{n}_{\Gamma}\right) \mathrm{d} S \approx \int_{\Gamma} \sum_{s=1}^{d} \mathbf{f}_{s}(\mathbf{w})\left(\boldsymbol{n}_{\Gamma}\right)_{s} \mathrm{~d} S,
$$

where $\mathbf{H}$ is a numerical flux. For the construction of the numerical flux we use the properties (2) of $\mathbf{f}_{s}$. Let us define the matrix

$$
\mathbf{P}(\mathbf{w}, \boldsymbol{n}):=\sum_{s=1}^{d}\left(\mathbf{A}_{s}(\mathbf{w})-z_{s} \mathbf{I}\right) n_{s},
$$

where $\boldsymbol{n}=\left(n_{1}, \ldots, n_{d}\right), n_{1}^{2}+\ldots+n_{d}^{2}=1$. Then we have

$$
\mathbf{P}(\mathbf{w}, \boldsymbol{n}) \mathbf{w}:=\sum_{s=1}^{d} \mathbf{g}_{s}(\mathbf{w}) n_{s}
$$

It is possible to show that the matrix $\mathbf{P}$ is diagonalizable. It means that there exists a nonsingular matrix $\mathbf{T}=\mathbf{T}(\mathbf{w}, \boldsymbol{n})$ and a diagonal matrix $\Lambda=\Lambda(\mathbf{w}, \boldsymbol{n})$ such that

$$
\mathbf{P}=\mathbf{T} \Lambda \mathbf{T}^{-1}, \quad \Lambda=\operatorname{diag}\left(\lambda_{1}, \ldots, \lambda_{d+2}\right),
$$

where $\lambda_{i}=\lambda_{i}(\mathbf{w}, \boldsymbol{n})$ are eigenvalues of the matrix $\mathbf{P}$. Then we can define the "positive" and "negative" parts of the matrix $\mathbf{P}$ by

$$
\mathbf{P}^{ \pm}=\mathbf{T} \Lambda^{ \pm} \mathbf{T}^{-1}, \quad \Lambda^{ \pm}=\operatorname{diag}\left(\lambda_{1}^{ \pm}, \ldots, \lambda_{d+2}^{ \pm}\right),
$$

where $\lambda^{+}=\max (\lambda, 0), \lambda^{-}=\min (\lambda, 0)$. Using this concept, we introduce the so-called Vijayasundaram numerical flux

$$
\mathbf{H}\left(\mathbf{w}^{L}, \mathbf{w}^{R}, \boldsymbol{n}\right)=\mathbf{P}^{+}\left(\frac{\mathbf{w}^{L}+\mathbf{w}^{R}}{2}, \boldsymbol{n}\right) \mathbf{w}^{L}+\mathbf{P}^{-}\left(\frac{\mathbf{w}^{L}+\mathbf{w}^{R}}{2}, \boldsymbol{n}\right) \mathbf{w}^{R} .
$$

This numerical flux has suitable form for a linearization. Now we can define inviscid form in the following way:

$$
\begin{aligned}
& b_{h}\left(\overline{\mathbf{w}}_{h}, \mathbf{w}_{h}, \Phi_{h}, t\right):= \\
& -\sum_{K \in \mathcal{T}_{h}(t)} \int_{K} \sum_{s=1}^{d}\left(\mathbf{A}_{s}\left(\overline{\mathbf{w}}_{h}\right)-z_{s}(t) \mathbf{I}\right) \mathbf{w}_{h} \cdot \frac{\partial \Phi_{h}}{\partial x_{s}} \mathrm{~d} \boldsymbol{x}
\end{aligned}
$$




$$
\begin{aligned}
& +\sum_{\Gamma \in \mathcal{F}_{h}^{I}(t)} \int_{\Gamma}\left(\mathbf{P}^{+}\left(\left\langle\overline{\mathbf{w}}_{h}\right\rangle, \boldsymbol{n}_{\Gamma}\right) \mathbf{w}_{h}^{L}+\mathbf{P}^{-}\left(\left\langle\overline{\mathbf{w}}_{h}\right\rangle, \boldsymbol{n}_{\Gamma}\right) \mathbf{w}_{h}^{R}\right) \cdot\left[\Phi_{h}\right] \mathrm{d} S \\
& +\sum_{\Gamma \in \mathcal{F}_{h}^{B}(t)} \int_{\Gamma}\left(\mathbf{P}^{+}\left(\left\langle\overline{\mathbf{w}}_{h}\right\rangle, \boldsymbol{n}_{\Gamma}\right) \mathbf{w}_{h}^{L}+\mathbf{P}^{-}\left(\left\langle\overline{\mathbf{w}}_{h}\right\rangle, \boldsymbol{n}_{\Gamma}\right) \overline{\mathbf{w}}_{h}^{R}\right) \cdot \Phi_{h} \mathrm{~d} S
\end{aligned}
$$

The boundary state $\overline{\mathbf{w}}_{h}^{R}$ is evaluated with the aid of the local linearized Riemann problem described in [4].

For the discretization of the viscous terms we use the property (3) and get the viscous form

$$
\begin{aligned}
& a_{h}\left(\overline{\mathbf{w}}_{h}, \mathbf{w}_{h}, \Phi_{h}, t\right):= \\
& +\sum_{K \in \mathcal{T}_{h}(t)} \int_{K} \sum_{s=1}^{d} \sum_{k=1}^{d} \mathbf{K}_{s, k}\left(\overline{\mathbf{w}}_{h}\right) \frac{\partial \mathbf{w}_{h}}{\partial x_{k}} \cdot \frac{\partial \Phi_{h}}{\partial x_{s}} \mathrm{~d} \boldsymbol{x} \\
& -\sum_{\Gamma \in \mathcal{F}_{h}^{I}(t)} \int_{\Gamma} \sum_{s=1}^{d}\left\langle\sum_{k=1}^{d} \mathbf{K}_{s, k}\left(\overline{\mathbf{w}}_{h}\right) \frac{\partial \mathbf{w}_{h}}{\partial x_{k}}\right\rangle\left(\boldsymbol{n}_{\Gamma}\right)_{s} \cdot\left[\Phi_{h}\right] \mathrm{d} S \\
& -\sum_{\Gamma \in \mathcal{F}_{h}^{B}(t)} \int_{\Gamma} \sum_{s=1}^{d} \sum_{k=1}^{d} \mathbf{K}_{s, k}\left(\overline{\mathbf{w}}_{h}\right) \frac{\partial \mathbf{w}_{h}}{\partial x_{k}}\left(\boldsymbol{n}_{\Gamma}\right)_{s} \cdot \Phi_{h} \mathrm{~d} S \\
& -\Theta \sum_{\Gamma \in \mathcal{F}_{h}^{I}(t)} \int_{\Gamma} \sum_{s=1}^{d}\left\langle\sum_{k=1}^{d} \mathbf{K}_{k, s}^{T}\left(\overline{\mathbf{w}}_{h}\right) \frac{\partial \Phi_{h}}{\partial x_{k}}\right\rangle\left(\boldsymbol{n}_{\Gamma}\right)_{s} \cdot\left[\mathbf{w}_{h}\right] \mathrm{d} S \\
& -\Theta \sum_{\Gamma \in \mathcal{F}_{h}^{B}(t)} \int_{\Gamma} \sum_{s=1}^{d} \sum_{k=1}^{d} \mathbf{K}_{k, s}^{T}\left(\overline{\mathbf{w}}_{h}\right) \frac{\partial \Phi_{h}}{\partial x_{k}}\left(\boldsymbol{n}_{\Gamma}\right)_{s} \cdot \mathbf{w}_{h} \mathrm{~d} S
\end{aligned}
$$

We set $\Theta=1$ or $\Theta=0$ or $\Theta=-1$ and get the so-called symmetric version (SIPG) or incomplete version (IIPG) or nonsymetric version (NIPG), respectively, of the discretization of viscous terms.

Further, we define the interior and boundary penalty form $J_{h}^{\sigma}$ and the right-hand side form $l_{h}$ in the following way:

$$
\begin{aligned}
& J_{h}^{\sigma}\left(\mathbf{w}_{h}, \Phi_{h}, t\right):=\sum_{\Gamma \in \mathcal{F}_{h}^{I}(t)} \int_{\Gamma} \sigma\left[\mathbf{w}_{h}\right] \cdot\left[\Phi_{h}\right] \mathrm{d} S \\
& +\sum_{\Gamma \in \mathcal{F}_{h}^{B}(t)} \int_{\Gamma} \sigma \mathbf{w}_{h} \cdot \Phi_{h} \mathrm{~d} S, \\
& l_{h}\left(\overline{\mathbf{w}}_{h}, \Phi_{h}, t\right):=\sum_{\Gamma \in \mathcal{F}_{h}^{B}(t)} \int_{\Gamma} \sigma \mathbf{w}_{B} \cdot \Phi_{h} \mathrm{~d} S \\
& -\Theta \sum_{\Gamma \in \mathcal{F}_{h}^{B}(t)} \int_{\Gamma} \sum_{s=1}^{d} \sum_{k=1}^{d} \mathbf{K}_{k, s}^{T}\left(\overline{\mathbf{w}}_{h}\right) \frac{\partial \Phi_{h}}{\partial x_{k}}\left(\boldsymbol{n}_{\Gamma}\right)_{s} \cdot \mathbf{w}_{B} \mathrm{~d} S .
\end{aligned}
$$

where $\left.\sigma\right|_{\Gamma}=\frac{\mu C_{W}}{d(\Gamma)}$, where $d(\Gamma)$ is the diameter of $\Gamma \in \mathcal{F}_{h}(t)$ and $C_{W}>0$ is a suitable sufficiently large constant. The boundary state $\mathbf{w}_{B}$ is defined on the basis of the Dirichlet boundary conditions and extrapolation:

$$
\begin{array}{ll}
\mathbf{w}_{B}=\left(\rho_{D}, \rho_{D} v_{D 1}, \ldots, \rho_{D} v_{D d}, c_{v} \rho_{D} \theta_{\Gamma}^{L}+\frac{1}{2} \rho_{D}\left|\boldsymbol{v}_{D}\right|^{2}\right) & \text { on } \Gamma_{I}, \\
\mathbf{w}_{B}=\mathbf{w}_{\Gamma}^{L} \quad \text { on } \Gamma_{O}, & \\
\mathbf{w}_{B}=\left(\rho_{\Gamma}^{L}, \rho_{\Gamma}^{L} z_{D 1}, \ldots, \rho_{\Gamma}^{L} z_{D d}, c_{v} \rho_{\Gamma}^{L} \theta_{\Gamma}^{L}+\frac{1}{2} \rho_{\Gamma}^{L}\left|z_{D}\right|^{2}\right) & \text { on } \Gamma_{W}(t) .
\end{array}
$$

In the vicinity of discontinuities or steep gradients nonphysical oscillations can appear in the approximate solu- tion. In order to overcome this difficulty we employ artificial viscosity forms, see [4]. They are based on the discontinuity indicator

$$
g(t)(K):=\frac{1}{d(K)|K|^{3 / 4}} \int_{\partial K}\left[\bar{\rho}_{h}\right]^{2} d S, \quad K \in \mathcal{T}_{h}(t),
$$

where $\left[\bar{\rho}_{h}\right]$ is the jump of the function $\bar{\rho}_{h}$ (= the first component of the vector function $\overline{\mathbf{w}}_{h}$ ) on the boundary $\partial K$, $d(K)$ denotes the diameter of $K$ and $|K|$ denotes the area of the element $K$. Then we define the discrete discontinuity indicator

$$
\begin{array}{ll}
G(t)(K):=0 & \text { if } g(t)(K)<1, \\
G(t)(K):=1 & \text { if } g(t)(K) \geq 1, K \in \mathcal{T}_{h}(t),
\end{array}
$$

and the artificial viscosity forms

$$
\begin{aligned}
& \tilde{\beta}_{h}\left(\overline{\mathbf{w}}_{h}, \mathbf{w}_{h}, \Phi_{h}, t\right):= \\
& v_{1} \sum_{K \in \mathcal{T}_{h}(t)} d(K) G(t)(K) \int_{K} \nabla \mathbf{w}_{h} \cdot \nabla \Phi_{h} \mathrm{~d} \boldsymbol{x}, \\
& \tilde{J}_{h}\left(\overline{\mathbf{w}}_{h}, \mathbf{w}_{h}, \Phi_{h}, t\right):= \\
& v_{2} \sum_{\Gamma \in \mathcal{F}_{h}^{I}(t)} \frac{1}{2}\left(G(t)\left(K_{\Gamma}^{L}\right)+G(t)\left(K_{\Gamma}^{R}\right)\right) \int_{\Gamma}\left[\mathbf{w}_{h}\right] \cdot\left[\Phi_{h}\right] \mathrm{d} S,
\end{aligned}
$$

with constants $v_{1}$ and $v_{2}$.

All these forms are linear with respect to $\mathbf{w}_{h}$ and nonlinear with respect to $\overline{\mathbf{w}}_{h}$.

\subsection{Time discretization by the BDF method of the flow problem}

Let $0=t_{0}<t_{1}<\ldots<t_{M}=T$ be a partition of the interval $[0, T]$, and define the time step $\tau_{m}=t_{m}-t_{m-1}, m=1, \ldots, M$. For a time instant $t_{m}$ we use the approximation $\mathbf{w}\left(t_{m}\right) \approx \mathbf{w}_{h}^{m}$, defined in $\Omega_{h}\left(t_{m}\right)$. Moreover we set $\hat{\mathbf{w}}_{h}^{l}=\mathbf{w}_{h}^{l}\left(\mathcal{A}_{t_{l}}\left(\mathcal{A}_{t_{m}}^{-1}(x)\right)\right)$, $x \in \Omega_{h}\left(t_{m}\right), l<m$. Then we approximate the ALE derivative at time $t_{m}$ by the backward finite difference formula (BDF) of order $\mathrm{q}$

$$
\frac{D^{\mathcal{A}} \mathbf{w}_{h}}{D t}\left(t_{m}\right) \approx \alpha_{0} \mathbf{w}_{h}^{m}+\sum_{l=1}^{q} \alpha_{l} \hat{\mathbf{w}}_{h}^{m-l},
$$

where the coefficients $\alpha_{l}, l=0, . ., q$, depend on $\tau_{m-l}, l=$ $0, . ., q-1$. In the case $m<q$ we set $q:=m$. For the nonlinear parts of the forms we employ the extrapolation $\overline{\mathbf{w}}_{h}^{m}$

$$
\overline{\mathbf{w}}_{h}^{m}=\sum_{l=1}^{q} \beta_{l} \hat{\mathbf{w}}_{h}^{m-l}
$$

where coefficients $\beta_{l}, l=1, \ldots, q$, depend on $\tau_{m-l}, l=0, . ., q-$ 1 . In the case $m<q$ we set $q:=m$. Expression of the coefficients $\alpha_{l}, \beta_{l}$ of order $q \leq 3$ can be found in [1] or [2]. Finally, we set

$$
(\varphi, \psi)_{t}=\int_{\Omega_{h}(t)} \varphi \psi \mathrm{d} x
$$

We say that the function $\mathbf{w}_{h}^{m} \in \mathbf{S}_{h}^{p}\left(t_{m}\right)$ is the approximate solution of the problem (1) obtained by the BDF method, 
if it satisfies the conditions

$$
\begin{aligned}
& \left(\alpha_{0} \mathbf{w}_{h}^{m}+\sum_{l=1}^{q} \alpha_{l} \hat{\mathbf{w}}_{h}^{m-l}, \Phi_{h}\right)_{t_{m}}+\left(\left(z\left(t_{m}\right) \cdot \nabla\right) \mathbf{w}_{h}^{m}, \Phi_{h}\right)_{t_{m}} \\
& +a_{h}\left(\overline{\mathbf{w}}_{h}^{m}, \mathbf{w}_{h}^{m}, \Phi_{h}, t_{m}\right)+b_{h}\left(\overline{\mathbf{w}}_{h}^{m}, \mathbf{w}_{h}^{m}, \Phi_{h}, t_{m}\right) \\
& +\tilde{\beta}_{h}\left(\overline{\mathbf{w}}_{h}^{m}, \mathbf{w}_{h}^{m}, \Phi_{h}, t_{m}\right)+\tilde{J}_{h}\left(\overline{\mathbf{w}}_{h}^{m}, \mathbf{w}_{h}^{m}, \Phi_{h}, t_{m}\right) \\
& +J_{h}^{\sigma}\left(\mathbf{w}_{h}^{m}, \Phi_{h}, t_{m}\right) \\
= & l_{h}\left(\overline{\mathbf{w}}_{h}^{m}, \Phi_{h}, t_{m}\right) \quad \forall \Phi_{h} \in \mathbf{S}_{h}^{p}\left(t_{m}\right),
\end{aligned}
$$

where $\mathbf{w}_{h}^{0}$ is $\mathbf{S}_{h}^{p}\left(t_{0}\right)$-approximation of $\mathbf{w}^{0}$.

\subsection{Full space-time DGM discretization}

Another way how to construct a method of high-order accuracy both in space and time is the full space-time discontinuous Galerkin (ST-DG) method. We again consider a partition $0=t_{0}<t_{1}<\ldots<t_{M}=T$ of the interval $[0, T]$ and denote $I_{m}=\left(t_{m-1}, t_{m}\right), \tau_{m}=t_{m}-t_{m-1}$ for $m=1, \ldots, M$. We define the space $\mathbf{S}_{h, \tau}^{p, q}=\left(S_{h, \tau}^{p, q}\right)^{d+2}$, where

$$
S_{h, \tau}^{p, q}:=\left\{\phi ;\left.\phi\right|_{I_{m}}=\sum_{i=0}^{q} \zeta_{i} \phi_{i} \text {, where } \phi_{i} \in S_{h}^{p}(t), \zeta_{i} \in P^{q}\left(I_{m}\right)\right\} .
$$

with integers $p, q \geq 1$. $P^{q}\left(I_{m}\right)$ denotes the space of all polynomials in $t$ on $I_{m}$ of degree $\leq q$. Moreover for $\Phi \in \mathbf{S}_{h, \tau}^{p, q}$ we introduce the following notation:

$$
\begin{aligned}
& \Phi_{m}^{ \pm}=\Phi\left(t_{m}^{ \pm}\right)=\lim _{t \rightarrow t_{m \pm}} \Phi(t), \\
& \{\Phi\}_{m}=\Phi_{m}^{+}-\Phi_{m}^{-} .
\end{aligned}
$$

Approximate solution $\mathbf{w}_{h \tau}$ of the problem will be sought in the space $\mathbf{S}_{h, \tau}^{p, q}$. Since the functions of this space are in general discontinuous in time, we ensure the connection between $I_{m-1}$ and $I_{m}$ by the penalty term in time

$$
\left(\left\{\mathbf{w}_{h \tau}\right\}_{m-1}, \Phi_{h \tau}\left(t_{m-1}^{+}\right)\right)_{t_{m-1}}
$$

The initial state $\mathbf{w}_{h \tau}$ is included by the $L^{2}\left(\Omega_{h}\left(t_{0}\right)\right)$-projection of $\mathbf{w}^{0}$ on $\mathbf{S}_{h}^{p}\left(t_{0}\right)$ :

$$
\left(\mathbf{w}_{h \tau}\left(t_{0}^{+}\right), \Phi_{h \tau}\left(t_{0}^{+}\right)\right)_{t_{0}}=\left(\mathbf{w}^{0}, \Phi_{h \tau}\left(t_{0}^{+}\right)\right)_{t_{0}} \quad \forall \Phi_{h \tau} \in \mathbf{S}_{h, \tau}^{p, q} .
$$

Similary as in Section 3.2 we introduce a suitable linearization. We can use two possibilities.

1) We put $\overline{\mathbf{w}}_{h \tau}(t):=\mathbf{w}_{h}\left(t_{m-1}^{-}\right)$for $t \in I_{m}$.

2) We prolong the solution from the time interval $I_{m-1}$ to the time interval $I_{m}$.

We say that a function $\mathbf{w}_{h \tau} \in \mathbf{S}_{h, \tau}^{p, q}$ is the approximate solution of the problem (1) obtained by the ST-DG method, if it satisfies the following conditions

$$
\begin{aligned}
& \sum_{m=1}^{M} \int_{I_{m}}\left(\left(\frac{D^{\mathcal{A}} \mathbf{w}_{h \tau}}{D t}, \Phi_{h \tau}\right)_{t}+\left((\mathbf{z}(t) \cdot \nabla) \mathbf{w}_{h \tau}, \Phi_{h \tau}\right)_{t}\right) \mathrm{d} t \\
& +\sum_{m=1}^{M} \int_{I_{m}}\left(a_{h}\left(\overline{\mathbf{w}}_{h \tau}, \mathbf{w}_{h \tau}, \Phi_{h \tau}, t\right)+b_{h}\left(\overline{\mathbf{w}}_{h \tau}, \mathbf{w}_{h \tau}, \Phi_{h \tau}, t\right)\right) \mathrm{d} t \\
& +\sum_{m=1}^{M} \int_{I_{m}}\left(\tilde{\beta}_{h}\left(\overline{\mathbf{w}}_{h \tau}, \mathbf{w}_{h \tau}, \Phi_{h \tau}, t\right)+\tilde{J}_{h}\left(\overline{\mathbf{w}}_{h \tau}, \mathbf{w}_{h \tau}, \Phi_{h \tau}, t\right)\right) \mathrm{d} t
\end{aligned}
$$

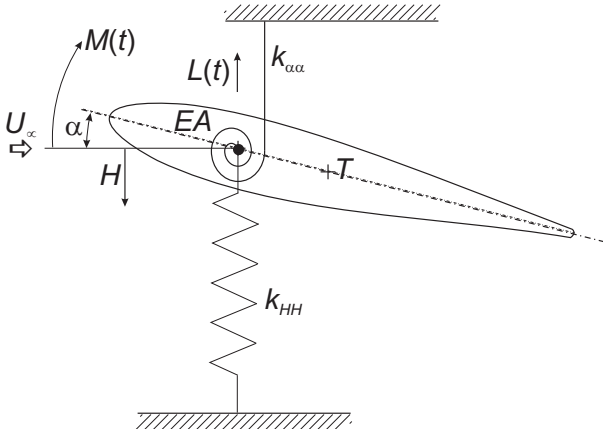

Fig. 1. The scheme of the vibrating airfoil.

$$
\begin{aligned}
& +\sum_{m=1}^{M} \int_{I_{m}} J_{h}^{\sigma}\left(\mathbf{w}_{h \tau}, \Phi_{h \tau}, t\right) \mathrm{d} t \\
& +\sum_{m=2}^{M}\left(\left\{\mathbf{w}_{h \tau}\right\}_{m-1}, \Phi_{h \tau}\left(t_{m-1}^{+}\right)\right)_{t_{m-1}}+\left(\mathbf{w}_{h \tau}\left(t_{0}^{+}\right), \Phi_{h \tau}\left(t_{0}^{+}\right)\right)_{t_{0}} \\
& =\sum_{m=1}^{M} \int_{I_{m}} l_{h}\left(\overline{\mathbf{w}}_{h \tau}, \Phi_{h \tau}, t\right) \mathrm{d} t+\left(\mathbf{w}^{0}, \Phi_{h \tau}\left(t_{0}^{+}\right)\right)_{t_{0}} \quad \forall \Phi_{h \tau} \in \mathbf{S}_{h, \tau}^{p, q} .
\end{aligned}
$$

\subsection{Equations for the moving airfoil}

We shall simulate the motion of a profile in $2 \mathrm{D}$ with two degrees of freedom: $H$ - displacement of the profile in the vertical direction (positively oriented downwards) and $\alpha$ - the rotation of the profile around the so-called elastic axis(positively oriented clockwise), see Figure 1. The motion of the profile is described by the system of ordinary differential equations

$$
\begin{aligned}
m \ddot{H}+S_{\alpha} \ddot{\alpha}+k_{H H} H & =-L(t), \\
S_{\alpha} \ddot{H}+I_{\alpha} \ddot{\alpha}+k_{\alpha \alpha} \alpha & =M(t),
\end{aligned}
$$

where we use the following notation: $m$ - mass of the airfoil, $L(t)$ - aerodynamic lift force, $M(t)$ - aerodynamic torsional moment, $S_{\alpha}$ - static moment of the airfoil around the elastic axis (EA), $k_{H H}$ - bending stiffness, $k_{\alpha \alpha}$ - torsional stiffness. We define $L(t)$ and $M(t)$ by the terms

$$
\begin{aligned}
L(t) & :=-l \int_{\Gamma_{W}(t)} \sum_{j=1}^{2} \tau_{2 j} n_{j} \mathrm{~d} S, \\
M(t) & :=l \int_{\Gamma_{W}(t)} \sum_{i, j=1}^{2} \tau_{i j} n_{j}(-1)^{i}\left(x_{1+\delta_{1 i}}-x_{1+\delta_{1 i}}^{E A}\right) \mathrm{d} S,
\end{aligned}
$$

where $l$ is the depth of the airfoil, $x_{i}^{E A}$ are the coordinates of the elastic axis and $\tau_{i j}:=-p \delta_{i j}+\tau_{i j}^{V}$ are the components of the stress tensor. For the derivation of the system (4), see e.g. [5].

The system (4) is transformed to a first-order system and solved by the fourth-order Runge-Kutta method together with the discrete flow problem.

\section{Numerical experiments}

We performed numerical simulations in 2D for the profile NACA0012 with the following data and initial conditions: $m=0.086622 \mathrm{~kg}, S_{a}=-0.000779673 \mathrm{~kg} \mathrm{~m}$, 


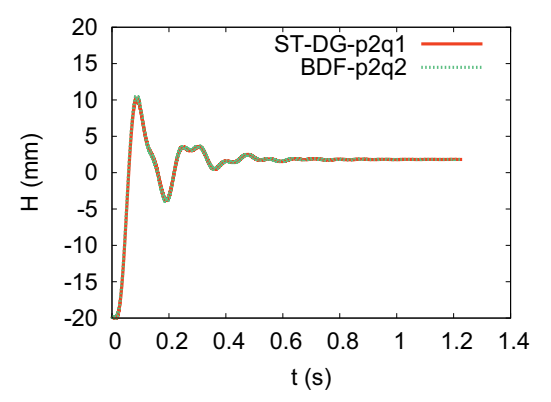

Fig. 2. Displacement for far-field velocity $20 \mathrm{~ms}^{-1}$.

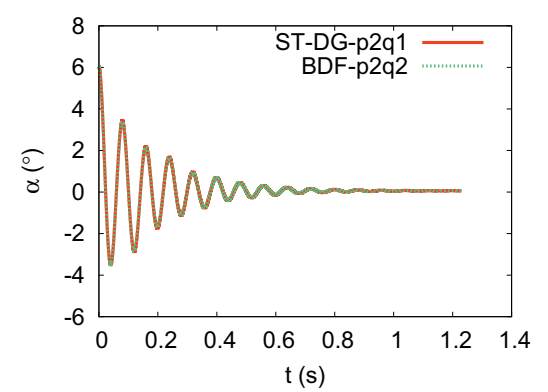

Fig. 3. Rotation angel for far-field velocity $20 \mathrm{~ms}^{-1}$.

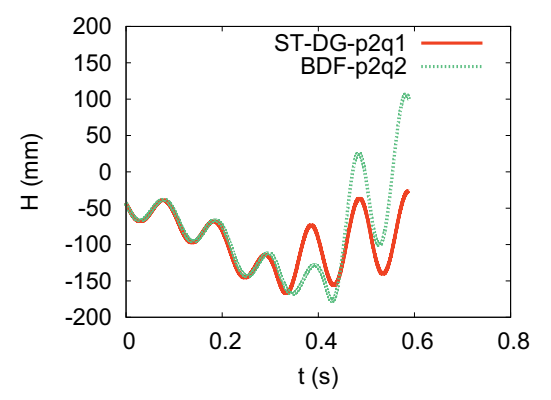

Fig. 4. Displacement for far-field velocity $40 \mathrm{~ms}^{-1}$.

$I_{a}=0.000487291 \mathrm{~kg} \mathrm{~m}^{-2}, k_{H H}=105.109 \mathrm{Nm}^{-1}, k_{\alpha \alpha}=$ $3.696682 \mathrm{~N} \mathrm{~m} \mathrm{rad}^{-1}, \mu=1.72 \cdot 10^{-5} \mathrm{~kg} \mathrm{~m}^{-1} \mathrm{~s}^{-1}$, far-field pressure $p=101250 \mathrm{~Pa}$, airfoil depth $l=0.05 \mathrm{~m}$, airfoil length $c=0.3 \mathrm{~m}$, far-field density $\rho=1.225 \mathrm{~kg} \mathrm{~m}^{-3}$, Poisson adiabatic constant $\gamma=1.4$, specific heat $c_{v}=721.428$ $\mathrm{m}^{2} \mathrm{~s}^{-2} \mathrm{~K}^{-1}$, heat conduction coefficient $k=2.428 \cdot 10^{-2} \mathrm{~kg}$ $\mathrm{m} \mathrm{s}^{-2} \mathrm{~K}^{-1}, H(0)=-20 \mathrm{~mm}, \alpha(0)=6^{\circ}, \dot{H}(0)=\dot{\alpha}(0)=0$. For all numerical simulations we chose symmetric version of the viscous term (SIPG), the parameter $C_{W}=500$ for all $\Gamma \in \mathcal{F}_{h}(t)$ except for $\Gamma \subset \Gamma_{W}(t)$, where we set $C_{W}=5000$. The constants in the artificial viscosity forms were set $v_{1}=$ $v_{2}=0.1$. The time step was choosen $\tau=0.003299 \mathrm{c} /\left|\boldsymbol{v}_{\infty}\right|$, where $\left|\boldsymbol{v}_{\infty}\right|$ is the far-field velocity. We employed quadratic polynomials $(\mathrm{p}=2)$ for the space discretization. In the case of the BDF we used the second order approximation for the time discretization (BDF-p2q2). In the case of the STDG we used linear polynomials in time (ST-DG-p2q1). Far field-velocity was choose $20 \mathrm{~ms}^{-1}$ and $40 \mathrm{~ms}^{-1}$. In this cases Reynolds number is between $10^{5}$ and $10^{6}$. Figures 2-5 show the computed displacement and rotation of the profile obtained by both methods.

At the end we present an example of high-speed flow for far-field velocity $680 \mathrm{~ms}^{-1}$ and Reynolds number $10^{7}$. In this case we left the data and initial conditions the same except bending stiffness and torsional stiffness which were

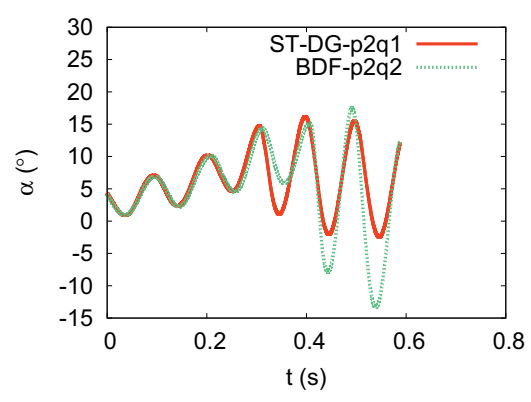

Fig. 5. Rotation angel for far-field velocity $40 \mathrm{~ms}^{-1}$.

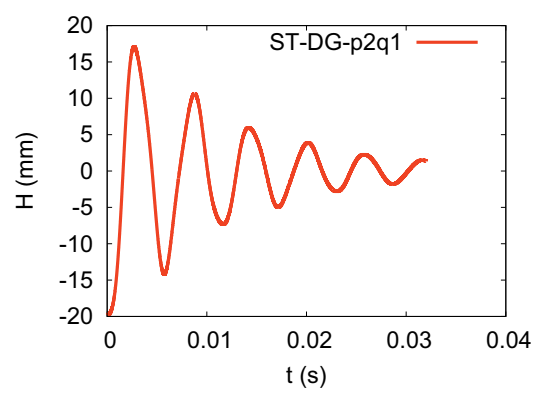

Fig. 6. Displacement for far-field velocity $680 \mathrm{~ms}^{-1}$.

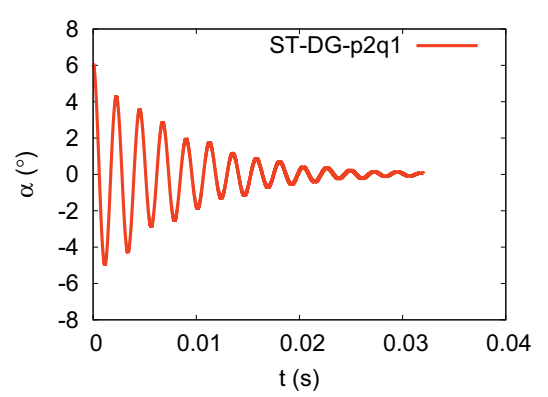

Fig. 7. Rotation angel for far-field velocity $680 \mathrm{~ms}^{-1}$.

chosen 1000 times higher. Figures 6-7 show the displacement and rotation of the profile obtained only by ST-DG, because for this regimes the BDF method became unstable. Figures 8 and 9 display distribution of the Mach number and pressure at time instant $t=0.00087 \mathrm{~s}$.

\section{Conclusion}

In this paper we dealt with the discontinuous Galerkin method for the numerical solution of compressible viscous flow. The applicability of the proposed method was demonstrated on the example of interaction of compressible viscous flow and a moving airfoil. Subject for the further work is including a turbulence model into the method.

\section{Acknowledgment}

This result originated with the support of Ministry of Industry and Trade of the Czech Republic for the long-term strategic development of the research organisation. The authors acknowledge this support. 

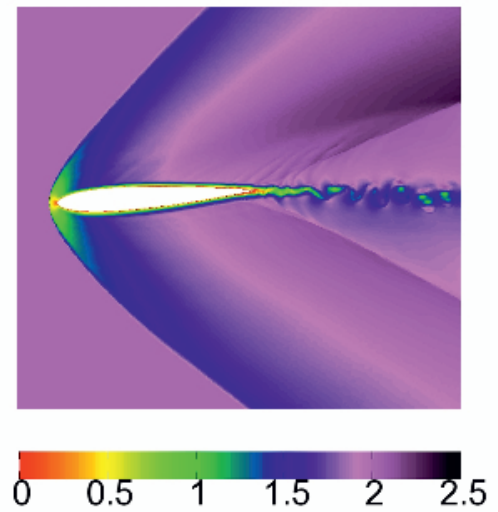

Fig. 8. The distribution of the Mach number at $t=0.00087 \mathrm{~s}$ for far-field velocity $680 \mathrm{~ms}^{-1}$.

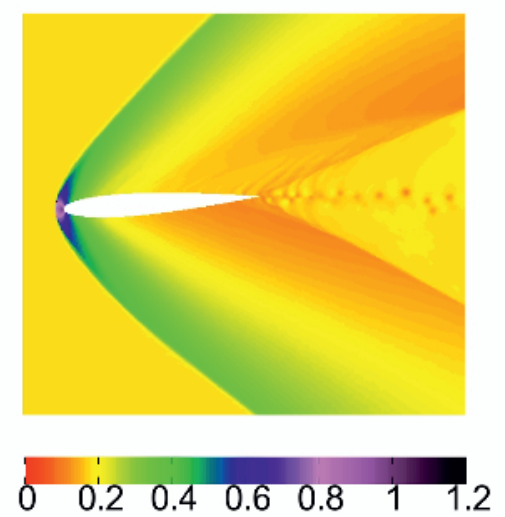

Fig. 9. The distribution of the pressure related to the quantity $\rho_{\infty}\left|\boldsymbol{v}_{\infty}\right|^{2}$, where $\rho_{\infty}$ and $\boldsymbol{v}_{\infty}$ mean far-field density and far-field velocity, at $t=0.00087 \mathrm{~s}$ for far-field velocity $680 \mathrm{~ms}^{-1}$.

\section{References}

1. Česenek J. Discontinuous Galerkin method for solving compressible viscous flow. Doctoral Thesis. Charles University, Faculty of Mathematics and Physics, Prague (2011).

2. Česenek J., Feistauer M., Kosik A. ZAMM - Z. Angew. Math. Mech. 93, 6 - 7, 387 - 402 (2013).

3. Dolejší V.: Commun. Comput. Phys., 4, 231-274 (2008).

4. Feistauer M., Felcman J., and Straškraba I.

Mathematical and Computational Methods for Compressible Flow. Clarendon Press, Oxford (2003).

5. Sváček P., Feistauer M., Horáček J. J. Fluids Struc., 23, 391-411 (2007). 